\title{
Tradução para o português e validação de conteúdo da Social Rhythm Metric-17 (SRM-17)
}

\section{Translation into Portuguese and content validation of the Social Rhythm Metric-17 (SRM-17)}

\author{
Regina Lopes Schimitt ${ }^{1}$, Maria Paz Loayza Hidalgo ${ }^{2}$
}

${ }^{1}$ Especialista, Psicologia Clínica. Psicóloga. Mestranda, Ciências Médicas, Faculdade de Medicina (FAMED), Universidade Federal do Rio Grande do Sul (UFRGS), Porto Alegre, RS. ${ }^{2}$ Professora, Departamento de Psiquiatria e Medicina Legal, FAMED, UFRGS. Laboratório de Cronobiologia, Hospital de Clínicas de Porto Alegre (HCPA), Porto Alegre, RS.

Suporte financeiro: Fundo de Incentivo à Pesquisa e Eventos do Hospital de Clínicas de Porto Alegre (FIPE-HCPA), Porto Alegre, RS.

\section{Resumo}

Objetivos: Traduzir para o português a Social Rhythm Metric-17 (SRM-17), escala que afere ritmo social, e realizar validação de conteúdo da versão produzida.

Método: Foi utilizada uma adaptação do método para validação de instrumentos que compreendeu as seguintes etapas: preparação, tradução, conciliação, retrotradução, revisão da retrotradução, avaliação de clareza, revisão de resultados da avaliação de clareza e finalização, prova de leitura e relatório final.

Resultados: A versão final brasileira manteve uma equivalência de itens com relação à primeira versão em inglês do instrumento original, incorporando melhorias. A transposição do instrumento alternativo criado pelo grupo de Cronobiologia Humana do Hospital de Clínicas de Porto Alegre (HCPA), Porto Alegre (RS), para a SRM-17 não mostrou discrepâncias significativas e revelou que, embora essa escala seja um instrumento genérico, é sensível às diferenças individuais quanto à aferição de ritmo social. A versão avaliada demonstrou um grau satisfatório de clareza e equivalência semântica.

Conclusão: Este trabalho apresenta uma versão adaptada à realidade brasileira de um instrumento específico para aferir ritmo social. O processo de adaptação transcultural deve efetivar-se com estudos de validação do instrumento final em uma amostra maior da população, nos quais também poderão ser avaliadas as equivalências operacional, de medida e funcional.

Descritores: Social Rhythm Metric, zeitgeber, cronobiologia, ritmo biológico, transtorno mental.

\begin{abstract}
Objectives: To translate the Social Rhythm Metric-17 (SRM-17), a scale that assesses social rhythm, into Portuguese and to validate the content of the Portuguese version.

Methods: An adaptation of the method for validation of instruments was used and it included the following stages: preparation, translation, reconciliation, back translation, revision of back translation, comprehension assessment, review of the results of comprehension assessment and completion, proofreading, and final report.

Results: The final Brazilian version has the same number of items as the first English version of the original instrument, and included some enhancements. The adaptation of the alternative instrument developed by the Human Chronobiology Group of Hospital de Clínicas de Porto Alegre, Porto Alegre, Brazil, to the SRM-17 did not show any significant discrepancies and revealed that, in spite of being a generic instrument, it is sensitive to the individual differences regarding the assessment of social rhythm. The Portuguese version showed a satisfactory level of understanding and semantic equivalence.

Conclusion: A version of an instrument to assess social rhythm adapted to the Brazilian reality is presented in this study. The cross-cultural adaptation process must be completed by validation studies of the final instrument in a larger population sample, which will also be able to evaluate the equivalences related to operation, measures, and functionality.

Keywords: Social rhythm metric, social Zeitgeber theory, chronobiology, biologic rhythm, mental disorder.
\end{abstract}

\section{Correspondência:}

Regina Lopes Schimitt, Rua Miguel Bauer, 1685, CEP 95600-000, Taquara, RS. Tel.: (51) 3542.2361, (51) 9155.3912. E-mail: regreg@tca.com.br

Não há conflitos de interesse associados à publicação deste artigo.

Copyright $(\subseteq$ Revista de Psiquiatria do Rio Grande do Sul - APRS

Recebido em 09/01/2009. Aceito em 17/02/2009. 


\section{Introdução}

O conceito de ritmo social considerado aqui se insere no campo teórico da cronobiologia, a ciência que estuda os ritmos biológicos, e faz fronteira com a psicologia do comportamento na medida em que é descrito como o ritmo determinado pelas interações sociais de um indivíduo ou imposto por convenções sociais.

No âmbito da cronobiologia, os agentes externos sincronizadores de ritmos biológicos denominam-se zeitgebers. $\mathrm{O}$ ritmo social, então, é dado pelos zeitgebers sociais. A importância de estudar esse ritmo, que não é um ritmo biológico, mas atua na sincronização de ritmos biológicos, deve-se ao seu possível papel na etiologia de transtornos de humor ${ }^{1}$.

A escala de ritmo social foi concebida em 1990 por pesquisadores da Universidade de Pittsburg ${ }^{2}$, Pittsburg, EUA, para estudar a associação entre rupturas no ritmo social e início de episódios de transtorno de humor. Sabe-se que o restabelecimento da regularidade no ritmo social de pessoas deprimidas auxilia em sua recuperação, mas o papel da ruptura do ritmo social na etiologia dos transtornos de humor ainda não foi suficientemente estudado.

A escala de ritmo social é um formulário autoaplicável que consiste em uma lista de 15 atividades genéricas fixas e duas opcionais, devendo, o sujeito, registrar ao final do dia, durante um determinado período de tempo, se foram ou não realizadas, o horário em que foram realizadas e a quantidade e qualidade de interações sociais intervenientes durante a realização das mesmas.

A escala apura os dois índices mais importantes, o Hit e o ALI, a partir de um algoritmo ${ }^{3-5}$. Se uma atividade ocorre mais de três vezes por semana no mesmo horário ou dentro de uma faixa temporal que abrange os 45 minutos anteriores e posteriores a uma hora calculada como habitual, ela é considerada um Hit. A quantidade de Hits representa a regularidade na vida de um indivíduo e é o índice mais importante da escala. $\mathrm{O}$ escore de regularidade não possui um ponto de corte, mas varia de um mínimo de 0 (nenhuma regularidade) a um máximo que depende do número de dias aferidos. Se, por exemplo, o período aferido compreende 7 dias, o escore máximo é 7; se ele for de 9 dias, o escore máximo é 9; e assim sucessivamente. A escala foi concebida para avaliar a regularidade em um período mínimo de 1 semana.

$\mathrm{O}$ índice ALI representa o volume de atividades diferentes realizadas no período, sendo calculado através da contagem simples de toda atividade realizada a cada dia. De acordo com Monk et al. ${ }^{6}$, um escore inferior a 59 inviabiliza a avaliação de regularidade. Portanto, o ALI pode variar de 59 (pouca atividade) a um máximo que depende do período aferido. Se, por exemplo, o período aferido compreende 7 dias e o sujeito engaja-se em todas as 17 atividades contempladas pela escala, então o escore máximo será 119 (17 x 7).

O presente estudo descreve o processo de tradução e validação de conteúdo da versão em português da Social Rhythm Metric-17 (SRM-17).

\section{Método}

Ainda não existe um consenso sobre o processo de tradução de instrumentos como escalas e questionários para pesquisa na área da saúde, embora já exista boa literatura sobre o assunto ${ }^{7-20}$. Estabeleceu-se como princípio norteador o modelo de equivalência proposto por Herdman et al..$^{10}$ no intuito de obter um instrumento equivalente à versão original dos pontos de vista conceitual, de item e semântico. No presente estudo, foi utilizada uma adaptação do método proposto por Wild et al. ${ }^{8}$ para validação de instrumentos, que compreendeu as seguintes etapas: 1) preparação; 2) tradução; 3) conciliação; 4) retrotradução; 5) revisão da retrotradução; 6) avaliação de clareza; 7) revisão de resultados da avaliação de clareza e finalização; 8) prova de leitura; e 9) relatório final.

\section{Preparação}

Nesta etapa, que incluiu a compreensão do conceito cronobiológico de ritmo social na cultura brasileira, o estabelecimento de um vínculo com o autor do instrumento e o recrutamento da equipe de trabalho, também se buscou a equivalência conceitual. Esta não se obtém apenas com a tradução de um instrumento, devendo-se, verificar também se o construto investigado tem o mesmo significado para ambas as culturas.

Após a obtenção da autorização do autor do instrumento para a realização do processo de tradução e o estabelecimento de um canal de comunicação com ele para o esclarecimento de dúvidas posteriores, procurou-se compreender o conceito cronobiológico de ritmo social na cultura brasileira mediante uma busca bibliográfica acerca do conceito em língua portuguesa e de versões brasileiras de instrumentos psicométricos de medida do mesmo construto. Essa pesquisa revelou que, apesar de ser observável como fenômeno, o conceito tinha recebido pouca atenção enquanto variável cronobiológica. Já sob as perspectivas antropológica e sociológica, ele tinha emergido como conceito estabelecido.

Essa interface com as ciências sociais sinalizou a inviabilidade de uma tradução literal do instrumento, pois determinar zeitgebers sociais, uma das funções da escala, significa apontar quantos e quais são os eventos de cultura capazes de interferir na ritmicidade de eventos biológicos, o que introduz diferenças referentes à particularidade da cultura em questão.

A inexistência, em língua portuguesa, de qualquer instrumento psicométrico para avaliação isolada de ritmo social levou ao desenvolvimento de um instrumento alternativo para medida dessa variável com o propósito de estabelecer categorias de atividade e, posteriormente, analisar a equivalência de conceitos.

Esse instrumento experimental foi chamado de Mapa Temporal de Atividades. A ideia foi discutida durante um curso de cronobiologia promovido pelo Programa de Pós-Graduação em Ciências Médicas da Faculdade de Medicina da Universidade Federal do Rio Grande do Sul (UFRGS), Porto Alegre (RS), do qual participaram 10 membros do grupo de Cronobiologia Humana do HCPA, Porto Alegre (RS). Durante 9 dias, essas 
pessoas preencheram uma planilha diária de atividades elaborada no programa Excel com todas as atividades realizadas durante o dia e os seus respectivos horários. Também informaram, mediante um sinal convencionado, se houve interação social ou não durante essas atividades. Os resultados obtidos nessa planilha foram transpostos para a primeira versão da escala de ritmo social SRM-17 para verificar que categorias apontadas no instrumento desenvolvido pela equipe não eram contempladas pela escala e quais eram as implicações dessas omissões.

Em seguida, formou-se um grupo de discussão composto por profissionais da área da saúde, antropologia e letras, bem como por estudantes da área da saúde.

\section{Tradução}

Para o processo de tradução, foram selecionadas duas versões em inglês do instrumento, uma mais antiga, que parecia mais adequada à realidade brasileira, e a versão mais recente estabelecida pelo autor (Anexos 1 e 2) ${ }^{3}$. Para cada uma dessas versões foram realizadas duas traduções para o português: uma delas feita por um profissional em Tradução advertido sobre os conceitos subjacentes ao instrumento, e outra por outro tradutor profissional denominado "ingênuo", ou seja, não informado acerca dos conceitos e não pertencente à área da saúde. De acordo com Beaton et al. ${ }^{11}$, é importante que um dos tradutores tenha essas características para que seja menos influenciado por objetivos acadêmicos e possa oferecer uma tradução mais aproximada da linguagem usada pela população. Foi realizada ainda uma terceira tradução por um membro da equipe responsável pelo projeto.

\section{Conciliação}

A equipe fez uma conciliação dessas traduções a fim de obter uma versão única para a retrotradução.

\section{Retrotradução}

O processo de retrotradução para o inglês, realizado por um profissional tradutor de língua materna inglesa, não familiarizado com a versão original do instrumento e não advertido acerca dos conceitos, ajudou a apontar discrepâncias entre as versões originais e as resultantes de todo o processo.

\section{Revisão da retrotradução}

As discrepâncias identificadas foram discutidas com o autor do projeto. Não pôde ser feita a harmonização junto a versões em outras línguas do instrumento por não existirem outras versões, de acordo com o autor.

\section{Avaliação de clareza e validação de conteúdo}

Nesta etapa, buscou-se a equivalência de item e semântica. A equivalência de item diz respeito ao quanto a seleção de itens é adequada, relevante e aceitável a ambas as culturas, a cultura fonte (cultura da língua em que é escrita a versão original do instrumento) e a cultura alvo (para cuja língua ele está sendo adaptado). A equivalência semântica diz respeito ao quanto se conserva de significado na tradução para outra língua. De acordo com Herdman et al. ${ }^{10,16}$, existem sete tipos de significado: 1) o significado referencial, que representa as ideias ou objetos a que uma palavra ou conjunto de palavras se referem; 2) o significado conotativo, que concerne à resposta emocional evocada por uma palavra; 3) o significado estilístico ou social, que concerne ao quanto uma palavra é apropriada num contexto específico condicionado a geografia, tempo, estilo, nível de formalidade, sexo, etc. e imprópria em outro contexto; 4) o significado afetivo, que se refere ao modo como as palavras usadas podem refletir sentimentos e pontos de vista; 5) o significado refletido, que diz respeito aos modos como é possível interpretar uma palavra (como algo pode soar engraçado ou ofensivo em diferentes contextos); 6) o significado colocativo, que diz respeito ao modo pelo qual uma palavra extraída de um contexto estrangeiro pode ganhar força ou significado extra; e, finalmente, 7) o significado temático, que concerne ao modo pelo qual um significado particular é dado a uma mensagem pela forma em que é construída a frase.

A validação de conteúdo não é determinada estatisticamente, mas a partir da análise da representatividade dos itens em relação aos conceitos e à relevância dos objetivos a serem medidos ${ }^{21}$.

A versão mais antiga do instrumento, que foi escolhida pela equipe por parecer mais adequada à realidade brasileira, foi submetida à avaliação de 25 pesquisadores da área de cronobiologia e de cinco avaliadores da área de psicometria. Esses 30 profissionais analisaram o grau de clareza de cada uma das 15 sentenças da versão traduzida e adaptada para o português do Brasil da escala de ritmo social por meio de escala analógica visual (VAS) de $10 \mathrm{~cm}^{22,23}$. As instruções dadas foram: "Descreva o quão clara está a questão colocando um traço perpendicular à linha abaixo da questão. Lembre que 0 significa 'nada claro' e 10 'absolutamente claro'”. Foram definidos como indicador de compreensão insuficiente valores abaixo do percentil 25 . A compreensão global do instrumento foi definida como a média aritmética das 15 questões objetivas que o compõem (Tabela 1).

\section{Revisão dos resultados da avaliação de clareza e fina- lização, prova de leitura e relatório final}

Os participantes também foram convidados a anotar críticas e sugestões que foram levadas em consideração no estabelecimento da versão definitiva do instrumento. A versão definitiva (Anexo 3), embora semelhante à primeira versão em inglês do instrumento, incorporou melhorias da versão mais recente. Por último, o instrumento passou por prova de revisão para a correção de possíveis erros que não tivessem sido detectados em algum ponto do processo 
Tabela 1 - Versão avaliada da escala de ritmo social com as médias e desvio padrão do grau de clareza de cada questão medido pela escala analógica visual $(\mathrm{n}=30)$

\begin{tabular}{|c|c|c|c|}
\hline Questões - versão final & Questões - versão avaliada & Média (cm) & DP \\
\hline 1. Saí da cama & 1. Saiu da cama & 8,19 & 2,636 \\
\hline $\begin{array}{l}\text { 2. Falei pela primeira vez com outra pessoa } \\
\text { (pessoalmente ou por telefone) }\end{array}$ & $\begin{array}{l}\text { 2. Primeira comunicação (pessoalmente } \\
\text { ou por telefone) com outra pessoa }\end{array}$ & 8,84 & 1,453 \\
\hline $\begin{array}{l}\text { 3. Ouvi rádio ou assisti TV (em casa, no carro, } \\
\text { no trabalho, ou em outro lugar) }\end{array}$ & $\begin{array}{l}\text { 3. Primeiro contato com TV ou rádio } \\
\text { (em casa, carro, trabalho ou outro lugar) }\end{array}$ & 8,65 & 2,110 \\
\hline 4. Tomei café da manhã & 4. Tomou café da manhã & 9,32 & 1,058 \\
\hline 5. Saí de casa & 5. Saiu de casa & 9,16 & 1,299 \\
\hline 6. Fiz exercício físico & 6. Fez exercício físico & 8,68 & 1,615 \\
\hline $\begin{array}{l}\text { 7. Comecei o trabalho (ou a escola, o trabalho } \\
\text { de casa, trabalho voluntário, etc.) }\end{array}$ & $\begin{array}{l}\text { 7. Começou o trabalho (ou atividade estudantil, } \\
\text { ou doméstica, ou trabalho voluntário, etc.) }\end{array}$ & 9,20 & 0,961 \\
\hline 8. Almocei & 8. Almoçou & 9,46 & 0,843 \\
\hline 9. Tirei uma soneca & 9. Parou para cochilar & 8,45 & 2,122 \\
\hline 10. Fiz um lanche & 10. Fez um lanche & 8,71 & 2,310 \\
\hline 11. Tomei uma bebida alcoólica & 11. Tomou bebida alcoólica & 9,12 & 1,094 \\
\hline 12. Jantei & 12. Jantou & 9,04 & 2,017 \\
\hline $\begin{array}{l}\text { 13. Fiz uma atividade de lazer } \\
\text { (assisti TV, fui ao cinema, li, etc.) }\end{array}$ & $\begin{array}{l}\text { 13. Iniciou uma atividade noturna de lazer } \\
\text { (assistir TV, ir ao cinema, ler, etc.) }\end{array}$ & 8,52 & 1,939 \\
\hline 14. Desliguei TV ou o rádio pela última vez & 14. Desligou a TV ou o rádio pela última vez & 8,63 & 1,472 \\
\hline 15. Fui para a cama & 15. Foi para a cama & 9,05 & 1,374 \\
\hline
\end{tabular}

$\mathrm{DP}=$ desvio padrão; VAS = escala analógica visual.

\section{Resultados e discussão}

Neste trabalho observamos que, quanto à equivalência conceitual, o que se compreende por ritmo social na população brasileira é equivalente à compreensão deste conceito na cultura do instrumento original, já que a transposição do instrumento alternativo criado pelo grupo de Cronobiologia Humana do HCPA para a SRM-17 não mostrou discrepâncias significativas e revelou que, embora essa escala seja um instrumento genérico, é sensível às diferenças individuais quanto à aferição de ritmo social. As discussões entre os profissionais e estudantes da área da saúde envolvidos na avaliação do instrumento sugeriram a necessidade de modificação de alguns itens com a finalidade de obter equivalência conceitual.

Quanto à equivalência de item, a versão final brasileira manteve uma equivalência com relação à primeira versão em inglês do instrumento original, incorporando melhorias da segunda, como é exposto nos Anexos 1, 2 e 3.

A Tabela 1 mostra que a versão avaliada demonstrou um grau satisfatório de clareza e equivalência semântica. No entanto, o grau de dispersão revelou a necessidade de adequar melhor a escala à compreensão dos brasileiros.

A seguir, são relacionadas as modificações realizadas a partir dos procedimentos de avaliação de equivalência conceitual, de item e semântica:

1. Os verbos ficaram conjugados no pretérito perfeito porque a escala deverá ser preenchida ao final do dia, quando já ocorreram as ações. Isso auxilia quem está respondendo a organizar temporalmente os eventos.
2. Os verbos foram mantidos em todas as frases para manter a coerência entre os itens.

3. Item 1, "saí da cama", mantido como nas duas versões.

4. Item 2 mantido como na primeira versão. Optou-se pela expressão "falei pela primeira vez com outra pessoa (pessoalmente ou por telefone)" em lugar de "primeiro contato (first contact)" ou "primeira comunicação (first communication)".

5. Item 3 mantido como na primeira versão. Optou-se pela sentença "ouvi rádio ou assisti TV (em casa, no carro, no trabalho, ou em outro lugar)" como tradução possível para "first listen to TV or radio (in home, car, at work, wherever)", a fim de especificar melhor as ações referentes a cada objeto.

6. Item 4, "tomei café da manhã", mantido como nas duas versões. A expressão "tomei café da manhã" substituiu as expressões "have morning beverage" e "have breakfast" por ter sido considerada mais adequada à realidade brasileira;

7. Item 5, "saí de casa pela primeira vez", mantido como na segunda versão.

8. Item 6, "fiz exercício físico (caminhada, ginástica, dança, etc.)", mantido como na primeira versão. Foram, porém, acrescentados exemplos de atividades de acordo com sugestão apresentada por pessoas que avaliaram a clareza da escala.

9. Item 7, "comecei o trabalho (ou a escola, o trabalho de casa, trabalho voluntário, cuidados com crianças ou outros membros da família etc)": como tradução 
para "start work (or school, housework, volunteer activities, etc.)" optou-se por "comecei o trabalho" em vez de "comecei a trabalhar" por ser mais fiel ao original sem comprometer a clareza. "Trabalho voluntário" foi escolhido em lugar de "atividade voluntária" por ser um termo que designa muito especificamente o trabalho exercido de forma não remunerada e não qualquer ato voluntário.

10. Item 8, "almocei”, mantido nessa posição, como na primeira versão.

11. Item 9, "tirei uma soneca à tarde"; mantido como na segunda versão, porém na posição da primeira versão.

12. No item 10, a expressão "comi ou bebi algo à tarde (marcar apenas a primeira vez)" substituiu "have a snack" e "have an alcoholic drink", da primeira versão, ou "have an evening snack/drink", da segunda versão. A solicitação de marcar apenas a primeira vez deve-se ao fato de esta ser uma atividade que pode se repetir muitas vezes ao longo do dia, problema apontado pelos avaliadores da escala.

13. Item 11, "cheguei em casa pela última vez", mantido como o item 16 da segunda versão.

14. Item 12, "jantei", mantido como nos itens 12 e 9 da primeira e segunda versão respectivamente.

15. Item 13 mantido como na primeira versão. Optouse pela expressão "fiz uma atividade de lazer (assisti TV, fui ao cinema, li etc)" como uma alternativa mais adequada para "Engage in evening leisure activities (watch TV, go to movie, read, etc.)".

16. Item 14, "desliguei a TV ou o rádio pela última vez", mantido como na primeira versão.

17. Itens 15 e 16 mantidos como na segunda versão.

18. Item 17: Optou-se por "foi para cama", em vez de "deitou-se", por considerar-se que a primeira sentença caracteriza melhor o encerramento do dia.

\section{Conclusões}

Neste estudo obteve-se a validade de conteúdo mediante a apreciação de pessoas de reconhecido saber tanto na área de cronobiologia quanto na de psicometria. Este trabalho apresenta uma versão adaptada à realidade brasileira de um instrumento específico para aferir ritmo social equivalente à versão original dos pontos de vista conceitual, de item e semântico. A não existência de um consenso estabelecido sobre a adaptação transcultural de instrumentos psicométricos dificulta a padronização do processo. Para o estudo dessa questão, optou-se, principalmente, pelas abordagens de Wild et al. ${ }^{8}$ e Herdman et al. ${ }^{10}$ devido à abrangência de seus critérios. O processo de adaptação transcultural deve efetivarse com estudos de validação do instrumento final em uma amostra maior da população, que permitam avaliar também as equivalências operacional, de medida e funcional.

\section{Agradecimentos}

Ao Dr. Thimoty Monk, da Universidade de Pittsburg. Ao Professor Menna-Barreto do grupo de Cronobiologia Humana da Universidade de São Paulo (USP), São Paulo (SP).

\section{Referências}

1. Marques N, Menna-Barreto L. Cronobiologia: princípios e aplicações. $3^{\mathrm{a}}$ ed. São Paulo: Edusp; 2003.

2. Monk TH, Flaherty JF, Frank E, Hoskinson K, Kupfer DJ. The Social Rhythm Metric. An instrument to quantify the daily rhythms of life. J Nerv Ment Dis. 1990;178(2):120-6.

3. Monk TH, Kupfer DJ, Frank E, Ritenour AM. The Social Rhythm Metric (SRM): measuring daily social rhythms over 12 weeks. Psychiatry Res. 1991;36(2):195-207.

4. Frank E, Swartz HA, Kupfer DJ. Interpersonal and social rhythm therapy: managing the chaos of bipolar disorder. Biol Psychiatry. 2000;48(6):593-604.

5. Monk TH, Frank E, Potts, JM, Kupfer DJ. A simple way to measure daily lifestyle regularity. J Sleep Res. 2002;11(3):183-90.

6. Monk TH, Petrie SR, Hayes AJ, Kupfer DJ. Regularity of daily life in relation to personality, age, gender, sleep quality and circadian rhythms. J Sleep Res. 1994;3(4):196-205

7. Moraes CL, Hasselmann MH, Reichenheim ME. Adaptação transcultural para o português do instrumento "Revised Conflict Tatics Scales (CTS2)" utilizado para identificar violência entre casais. Cad Saude Publica. 2002;18(1):167-76.

8. Wild D, Grove A, Martin M, Eremenco S, McElroy S, Verjee-Lorenz A, et al. Principles of good practice for the ttranslation and cultural adaptation process for Patient-Reported Outcomes (PRO) measures: report of the ISPOR Task Force for Translation and Cultural Adaptation. Value Health. 2005;8(2):94-104.

9. Hauck S, Schestatsky S, Terra L, Knijnik L, Sanchez P, Ceitlin LH. Adaptação transcultural para o português brasileiro do Parental Bonding Instrument (PBI). Rev Psiquiatr RS. 2006;28(2):61-8.

10. Herdman M, Fox-Rushby J, Badia X. A model of equivalence in the cultural adaptation of HRQoL instruments: the universalist approach. Qual Life Res. 1998;7(4):323-35

11. Beaton DE, Bombardier C, Guillemin F, Ferraz MB. Guidelines for the process of cross-cultural adaptation of self-report measures. Spine (Phila Pa 1976). 2000;25(24):3186-91.

12. Chang AM, Chau JP, Holroyd E. Translation of questionnaires and issues of equivalence. J Adv Nurs. 1999;29(2):316-22.

13. Fiszman A, Cabizuca M, Lanfredi C, Figueira I. A adaptação transcultural para o português do instrumento dissociative experiences scale para rastrear e quantificar os fenômenos dissociativos. Rev Bras Psiquiatr. 2004;26(3):164-73.

14. Reichenheim ME, Moraes CL. Operacionalização de adaptação transcultural de instrumentos de aferição usados em epidemiologia. Rev Saude Publica. 2007;41(4):665-73.

15. Jorge MR. Adaptação transcultural de instrumentos de pesquisa em saúde mental. Rev Psiquiatr Clin. 1998;25(5):233-9.

16. Hasselmann $\mathrm{MH}$, Reichenheim ME. Adaptação transcultural da versão em português da Conflict Tactics Scales Form R (CTS-1), usada para aferir violência no casal: equivalências semântica e de mensuração. Cad Saude Publica. 2003;19(4):1083-93.

17. Eremenco SL, Cella D, Arnold BJ. A comprehensive method for the translation and cross-cultural validation of health status questionnaires. Eval Health Prof. 2005;28(2):212-32.

18. Wang WL, Lee HL, Fetzer SJ. Challenges and strategies of instrument translation. West J Nurs Res. 2006;28(3):310-20.

19. Sperber AD. Translation and validation of study instruments for cross-cultural research. Gastroenterology. 2004;126(1 Suppl 1):S124-8.

20. Harb A, Caumo W, Hidalgo MP. Tradução de adaptação da versão brasileira do Night Eating Questionnaire (NEQ). Cad Saude Publica. 2008;24:1368-76.

21. Fachel JM, Camey S. Avaliação psicométrica: a qualidade das medidas e o entendimento dos dados. In: Cunha JA, ed. Psicodiagnóstico V. $5^{\text {a }}$ ed. Porto Alegre: Artmed; 2000. p. 158-70.

22. Clark P, Lavielle P, Martínez H. Learning from pain scales: patient perspective. J Rheumatol. 2003;30(7):1584-8.

23. Creamer P, Lethbridge-Cejku M, Hochberg MC. Determinants of pain severity in knee osteoarthritis: effect of demographic and psychosocial variables using 3 pain measures. J Rheumatol. 1999;26(8):1785-92. 
Anexo 1 - Social Rhythm Metric (SRM-17) - Primeira versão

\begin{tabular}{|c|c|c|c|c|c|c|c|c|}
\hline \multirow[b]{2}{*}{$\begin{array}{l}\text { Respondent } \\
\text { Day } \\
\text { Date }\end{array}$} & \multirow[b]{2}{*}{$\begin{array}{l}\text { Time (circle } \\
\text { AM or PM) }\end{array}$} & \multicolumn{7}{|c|}{$\begin{array}{l}\text { Persons involved } \\
\text { (fill in all that apply) }\end{array}$} \\
\hline & & 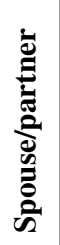 & 总 & 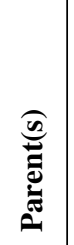 & $\frac{\widehat{n}}{0}$ & 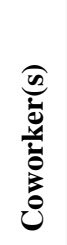 & 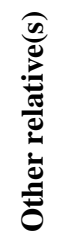 & $\frac{\mathscr{\Xi}}{4}$ \\
\hline 1. Get out of bed & $\begin{array}{l}\mathrm{AM} \\
\mathrm{PM}\end{array}$ & & & & & & & \\
\hline $\begin{array}{l}\text { 2. First communication (in person or by phone) } \\
\text { with another person }\end{array}$ & $\begin{array}{l}\mathrm{AM} \\
\mathrm{PM}\end{array}$ & & & & & & & \\
\hline 3. First listen to TV or radio (in home, car, at work, wherever) & $\begin{array}{l}\mathrm{AM} \\
\mathrm{PM}\end{array}$ & & & & & & & \\
\hline 4. Have breakfast & $\begin{array}{l}\mathrm{AM} \\
\mathrm{PM}\end{array}$ & & & & & & & \\
\hline 5. Leave home & $\begin{array}{l}\mathrm{AM} \\
\mathrm{PM}\end{array}$ & & & & & & & \\
\hline 6. Physical exercise & $\begin{array}{l}\mathrm{AM} \\
\mathrm{PM}\end{array}$ & & & & & & & \\
\hline 7. Start work (or school, housework, volunteer activities, etc.) & $\begin{array}{l}\mathrm{AM} \\
\mathrm{PM}\end{array}$ & & & & & & & \\
\hline 8. Have lunch & $\begin{array}{l}\mathrm{AM} \\
\mathrm{PM}\end{array}$ & & & & & & & \\
\hline 9. Take a nap & $\begin{array}{l}\mathrm{AM} \\
\mathrm{PM}\end{array}$ & & & & & & & \\
\hline 10.Have a snack & $\begin{array}{l}\mathrm{AM} \\
\mathrm{PM}\end{array}$ & & & & & & & \\
\hline 11. Have an alcoholic drink & $\begin{array}{l}\mathrm{AM} \\
\mathrm{PM}\end{array}$ & & & & & & & \\
\hline 12.Have dinner & $\begin{array}{l}\mathrm{AM} \\
\mathrm{PM}\end{array}$ & & & & & & & \\
\hline $\begin{array}{l}\text { 13. Engage in evening leisure activities (watch TV, } \\
\text { go to movie, read, etc.) }\end{array}$ & $\begin{array}{l}\text { AM } \\
\text { PM }\end{array}$ & & & & & & & \\
\hline
\end{tabular}




\section{Anexo 2 - Social Rhythm Metric (SRM - 17) - Segunda versão}

\begin{tabular}{|c|c|c|c|c|c|c|c|c|c|}
\hline \multicolumn{10}{|c|}{ Please fill this out at end of the day } \\
\hline \multirow[b]{3}{*}{ Activity } & \multirow[b]{3}{*}{ 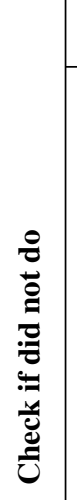 } & \multicolumn{3}{|c|}{ Time } & \multirow[b]{3}{*}{ 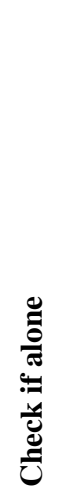 } & \multirow{2}{*}{\multicolumn{4}{|c|}{$\begin{array}{c}\text { People } \\
1=\text { just present } \\
2=\text { actively involved }\end{array}$}} \\
\hline & & & & & & & & & \\
\hline & & 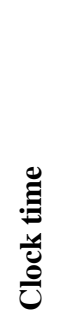 & ذ্ّ & $\dot{\Xi}$ & & 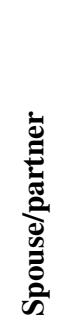 & 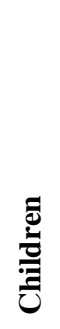 & 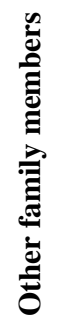 & 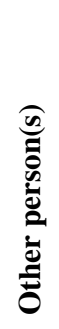 \\
\hline \multicolumn{10}{|c|}{ Out of bed } \\
\hline \multicolumn{10}{|c|}{ First contact (in person or by phone ) with another person } \\
\hline \multicolumn{10}{|c|}{ Have morning beverage } \\
\hline \multicolumn{10}{|c|}{ Have breakfast } \\
\hline \multicolumn{10}{|c|}{ Go outside for the first time } \\
\hline \multicolumn{10}{|c|}{ Start work, school, housework, volunteer activities, child or family care } \\
\hline \multicolumn{10}{|c|}{ Have lunch } \\
\hline \multicolumn{10}{|c|}{ Take an afternoon nap } \\
\hline \multicolumn{10}{|c|}{ Have dinner } \\
\hline \multicolumn{10}{|c|}{ Physical exercise } \\
\hline \multicolumn{10}{|c|}{ Have an evening snack/drink } \\
\hline \multicolumn{10}{|c|}{ Watch evening tv news program } \\
\hline \multicolumn{10}{|c|}{ Watch another tv program } \\
\hline \multicolumn{10}{|c|}{ Activity a........... } \\
\hline \multicolumn{10}{|c|}{ Activity b........... } \\
\hline \multicolumn{10}{|c|}{ Return home (last time) } \\
\hline Go to bed & & & & & & & & & \\
\hline
\end{tabular}




\section{Anexo 3 - Escala de ritmo social}

\begin{tabular}{|c|c|c|c|c|c|c|c|c|c|}
\hline \multicolumn{10}{|c|}{ Por favor, preencha esta tabela no fim do dia } \\
\hline \multicolumn{10}{|l|}{$\begin{array}{l}\text { Nome: } \\
\text { Dia da semana: } \\
\text { Data: }\end{array}$} \\
\hline \multirow[b]{3}{*}{ Atividade } & \multirow[b]{3}{*}{ 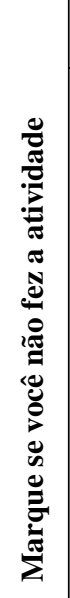 } & \multicolumn{3}{|c|}{ Horário } & & \multirow{3}{*}{ 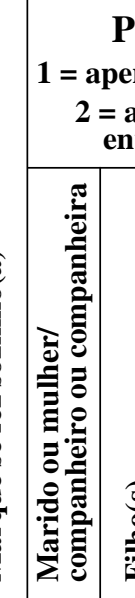 } & \multirow{2}{*}{\multicolumn{3}{|c|}{$\begin{array}{l}\text { Pessoas } \\
\text { enas presentes } \\
\text { ativamente } \\
\text { avolvidas }\end{array}$}} \\
\hline & & & Mar & que & & & & & \\
\hline & & 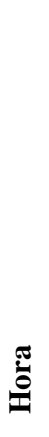 & 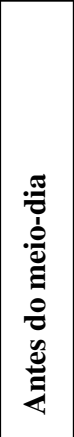 & 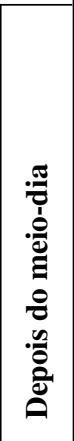 & 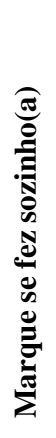 & & 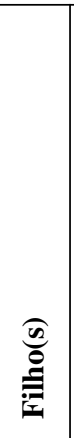 & 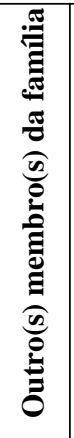 & 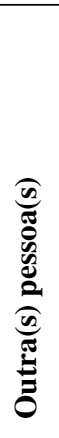 \\
\hline \multicolumn{10}{|l|}{ Saí da cama } \\
\hline \multicolumn{10}{|c|}{ Falei pela primeira vez com outra pessoa (pessoalmente ou por telefone) } \\
\hline \multicolumn{10}{|c|}{$\begin{array}{l}\text { Ouvi rádio ou assisti TV (em casa, no carro, no trabalho, } \\
\text { ou em outro lugar) }\end{array}$} \\
\hline \multicolumn{10}{|c|}{ Tomei café da manhã } \\
\hline \multicolumn{10}{|c|}{ Saí de casa pela primeira vez } \\
\hline \multicolumn{10}{|c|}{ Fiz exercício físico (caminhada, ginástica, dança, etc.) } \\
\hline \multicolumn{10}{|c|}{$\begin{array}{l}\text { Comecei o trabalho (ou a escola, o trabalho de casa, trabalho } \\
\text { voluntário, cuidados com crianças ou outros membros da família, etc.) }\end{array}$} \\
\hline \multicolumn{10}{|l|}{ Almocei } \\
\hline \multicolumn{10}{|c|}{ Tirei uma soneca à tarde } \\
\hline \multicolumn{10}{|c|}{ Comi ou bebi algo à tarde (marcar apenas a primeira vez) } \\
\hline \multicolumn{10}{|c|}{ Cheguei em casa pela última vez } \\
\hline \multicolumn{10}{|l|}{ Jantei } \\
\hline \multicolumn{10}{|c|}{ Fiz uma atividade de lazer (assisti TV, fui ao cinema, li, etc.) } \\
\hline \multicolumn{10}{|c|}{ Desliguei a TV ou o rádio pela última vez } \\
\hline \multicolumn{10}{|l|}{ Atividade a............ } \\
\hline \multicolumn{10}{|c|}{ Atividade b.......... } \\
\hline Fui para a cama & & & & & & & & & \\
\hline
\end{tabular}

